\title{
Early Life Stress as Factor for Use of Psychoactive Substances: Integrative Review
}

\section{Larissa Bessani Hidalgo Gimenez ${ }^{1}$, Renata Cristina de Campos Pereira Silveira², Danilo Caetano Albino da Silva ${ }^{3}$, Edilaine Cristina da Silva Gherardi-Donato ${ }^{{ }^{*}}$}

${ }^{1}$ Department of Psychiatric Nursing and Human Sciences, University of São Paulo at Ribeirão Preto College of Nursing, Ribeirao Preto, Brazil

${ }^{2}$ Departament of Fundamental Nursing, University of São Paulo at Ribeirão Preto College of Nursing, Ribeirao Preto, Brazil

${ }^{3}$ Itapevi Diagnostic Imaging Center, São Paulo, Brazil

Email: lari_gimenez@hotmail.com, ${ }^{*}$ nane@eerp.usp.br

How to cite this paper: Gimenez, L.B.H., Silveira, R.C.C.P., Silva, D.C.A. and Gherardi-Donato, E.C.S. (2016) Early Life Stress as Factor for Use of Psychoactive Substances: Integrative Review. Open Journal of Nursing, 6, 921-936.

http://dx.doi.org/10.4236/ojn.2016.611089

Received: August 5, 2016

Accepted: November 1, 2016

Published: November 4, 2016

Copyright $\odot 2016$ by authors and Scientific Research Publishing Inc. This work is licensed under the Creative Commons Attribution International License (CC BY 4.0).

http://creativecommons.org/licenses/by/4.0/

c) (i) Open Access

\begin{abstract}
Purpose: To identify, analyze and synthetize the scientific evidences that support Early Life Stress (ELS) diagnosis using the Childhood Trauma Questionnaire (CTQ), since it assumes the use of psychoactive substances later in adolescence and adulthood. Individuals that experienced some form of childhood stress may present, in adulthood, consequences that manifest into mental disorders such as problematic use of psychoactive substances. Methods: An integrative review of the literature on the subject in the databases: PubMed, Web of Science, LILACS and Psycinfo, regards studies that have been indexed in the last ten years (2003-2014). Combination of controlled and uncontrolled factors in the use and abuse of psychoactive substances, CTQ and ELS adapted to each database. Findings: The alcohol was the most used drug by the participating subjects of these research; mostly, the use of psychoactive drugs, started when they were still in adolescence; ELS and the later use of psychoactive drugs can be found more frequently in female; the lack of internal resources for dealing with stress in adulthood after ELS occurrence may have as a consequence the use of psychoactive drugs as a coping mechanism. Conclusions: It was possible to identify scientific evidences that support the ELS diagnosis, measured by the CTQ, as a determining factor for the use and abuse of alcohol and/or other psychoactive drugs in adolescence and adulthood. Implications: Investigate the ELS as a strategy to improve the therapeutic project of patients in nursing care, which will be built based on scientific evidence, so it can be more effective.
\end{abstract}

\section{Keywords}

Childhood Trauma Questionnaire, Early Life Stress, Evidence-Based Practice, Substance Abuse 


\section{Introduction}

A leaning towards psychological stress is the result of cognitive distortions and a physiological hyper reactivity before psychosocial demands that may be created due to hyper sensibility of the limbic system, which produces excessive catecholamines, testosterone and cortisol. Some individuals seem to have the tendency to stress, which can be the result of outside forces, and its effects are mediated by the ability to face stress, something learned mainly during childhood [1].

The early and cumulative (chronical) exposure to stress factors may result in neuroendocrine alterations and subjective behavioral changes. These stress factors tend to be involved in long-term process and hinder the development of the brain systems that are responsible for learning, motivation, work stress reductions and adaptive behavior [2].

Individuals that experienced some form of childhood stress may present, in adulthood, consequences that manifest into mental disorders such as: humor, anxiety, personality disorders and the problematic use of alcohol and other substances [3].

ELS experiences lived in childhood may be Physical Abuse, Sexual Abuse, Emotional Abuse, Physical Neglect and Emotional Neglect, according to Bernstein et al. (2003) [4].

Physical Abuse is when aggressions are committed by someone older with the risk of lesions, which are erroneously used to educate children by parent or guardians, resulting in body wounds that may lead to death.

Sexual Abuse is any contact or sexual behavior between a child and someone older, seeking to sexually stimulate the child or adolescent and/or using them to obtain sexual stimulation for someone or a third party.

Emotional Abuse is verbal aggressions that affect a child's wellbeing or moral integrity, or any conduct that humiliates, embarrasses or threatens the child. It also occurs when an adult constantly depreciates a child, blocking their efforts in self-acceptance and causing emotional grief.

Physical Neglect is when a parent or a responsible party fails in providing food, adequate clothing or other basic necessities such as: shelter, security and health supervision.

Emotional Neglect is when a guardian fails in providing basic emotional and psychological needs such as love support and motivation, also, when a child emotional necessity is not given, such as affection, cognitive and psychological support [4].

Stress during the early stages of life is a precursor of alcohol abuse and/or other drugs and dependence of such in adulthood. The risk/resilience relation to dependence on psychoactive drugs may be, in part, due to the interaction between genetic variations and environment stress factors, such as experiences with early types of abuse (sexual abuse during childhood, physical and emotional abuse, physical or emotional neglect) [5].

Psychoactive substance dependence may onset in individuals due to the influence of biological, psychological and social factors. Biological factor are associated with each individual's organism, the psychological are associated with personality, fears, anxiety 
and insecurity in facing everyday life situations. Social factors are associated with family, culture or, in other words, with the context in which the subject finds himself inserted in. The individual that receives attention, care and limits in an adequate manner are more equipped to grow into well-adjusted adults. Those who face negligence have a tendency to inappropriate behaviors and emotional hardship to solve conflicts [6].

There are many tools that can be used to investigate traumatic events in childhood, although a lot of these have low sensibility and trustability. Many forms of measuring traumas are focused solely on physical or sexual abuse, ignoring other forms of mistreatment, such as the emotional ones. Bernstein et al. (1997) created a self-applicable questionnaire with 70 items, called Childhood Trauma Questionnaire (CTQ). It was created using the literature associated with mistreatment and the tools of abuse and neglect during childhood in adults. This instrument investigates five traumatic components: Physical Abuse, Sexual Abuse, Emotional Abuse, Physical Neglect and Emotional Neglect. It also deals with a scale that controls minimizations/denial in the answers provided [7].

It is a tool focused on adolescents (up to twelve years old) and adults, with which are classified the frequency of twenty-eight questions related to situations that happened during childhood and measured by Likert's scale of five points. Factorial, exploratory and confirmatory analysis of the constructo (model by esctrutural equation) show that the 25 itens of the instrument (excluding 3 items of the minimization/negation scale) compose 5 distinct factors that match the 5 dimensions hypothesized for the instrument. The Childhood Trauma Questionnaire is a standardized selfreport inventory that measures the severity of five different types of childhood trauma and tendency to underreport maltreatment. Previous research on the CTQ has demonstrated its validity and reliability among clinical and non-clinical populations [7].

We decide to standardize our sample with this tool because the CTQ is a very commonly used tool, and can be considered the gold standard tool for research in the clinical and forensic fields of ELS [8].

The Integrative Review (IR) is a data collection method which contributes to the phase of the search for Evidence-Based Practice to construct a comprehensive literature analysis contributing to discussions on methods and results of research and experiments, and aims to deepen the understanding of certain phenomenon, based studies and previous evidence [9]. The Evidence-Based Practice comes from the EvidenceBased Medicine (EBM) had origin in the work of the British epidemiologist Archie Cochrane in 1972. It is an approach which defined a problem and carried out the search and evaluation of available evidence sore the subject, after the evidence is implemented in practice, and the results evaluated. This approach incorporates the evidence from research, along with the expertise of professional and customer preferences, enabling improved quality of care [11] [12].

\section{Purpose and Research Question}

The purpose of this integrative review was to Identify, Analyze and Synthetize scientific 
evidence that support the hypothesis that Early Life Stress (ELS), measured by the CTQ, may in fact result in the use and abuse of psychoactive substances in adolescence and adulthood.

We chose PICOT methodology to formulate the research question PICOT represents the acronym: $\mathrm{P}=$ Patient or Population; $\mathrm{I}=$ Intervention or Indicator; $\mathrm{C}=$ Comparison or Control; $\mathrm{O}=$ Outcomes; and $\mathrm{T}=$ Time [10].

In this review, the PICOT strategy was used in the following manner:

P-People that suffered Early Life Stress in childhood and used and abused of psychoactive drugs in adolescence and adulthood;

I-Having suffered Early Life Stress, measured by the CTQ;

C-(does not apply);

O-People that suffered Early Life Stress, measured by the CTQ, and eventually used and abused psychoactive substances in adolescence and adulthood;

$\mathrm{T}$-(does not apply).

And the research question was: Suffering ELS during childhood, measured by CTQ, is a determining factor in the use and abuse of psychoactive substances in adolescence and adulthood?

\section{Method}

\subsection{Sample Selection}

The sample inclusion criteria for this review were: primary research, search limited to the period between 2003 and 08/18/2014; languages (Portuguese, English and Spanish); quantitative and qualitative studies; Primary studies which include the use of psychoactive drugs in adolescence and adulthood as a result of ELS, measured by CTQ. Exclusion criteria: Review Articles; Secondary Articles; Guidelines; Editorials and Articles that researched ELS as the cause of other psychiatric clinical states that are not related to psychoactive substances.

The studies were selected from the following databases: PubMed, Web of Science, Latino-American and Caribbean Literature in Health Care Sciences (LILACS) and Psycinfo. PubMed: 298 references were found. After the appropriate filters, 244 remained. Web of Science: 96 references were found. After the appropriate filters, 90 remained. LILACS: 667 references were found. After the appropriate filters, 63 remained. Psycinfo: 53 references were found. After the appropriate filters, 42 remained. The total number of references found in the four databases was of 1114. Using filters regarding language, original articles and publications from 2003 to 2014, the total number of references was 439.

After removing articles that appeared more than once, due to different databases, the number of references left for this study was 359 . The titles and abstracts were evaluated according to relevance, and 157 studies were selected for a complete reading. After this integral reading, texts that didn't answer the research question were eliminated, resulting in eight articles left for this review. A flow diagram of study selection process was developed to illustrate the articles selection process (Figure 1). 


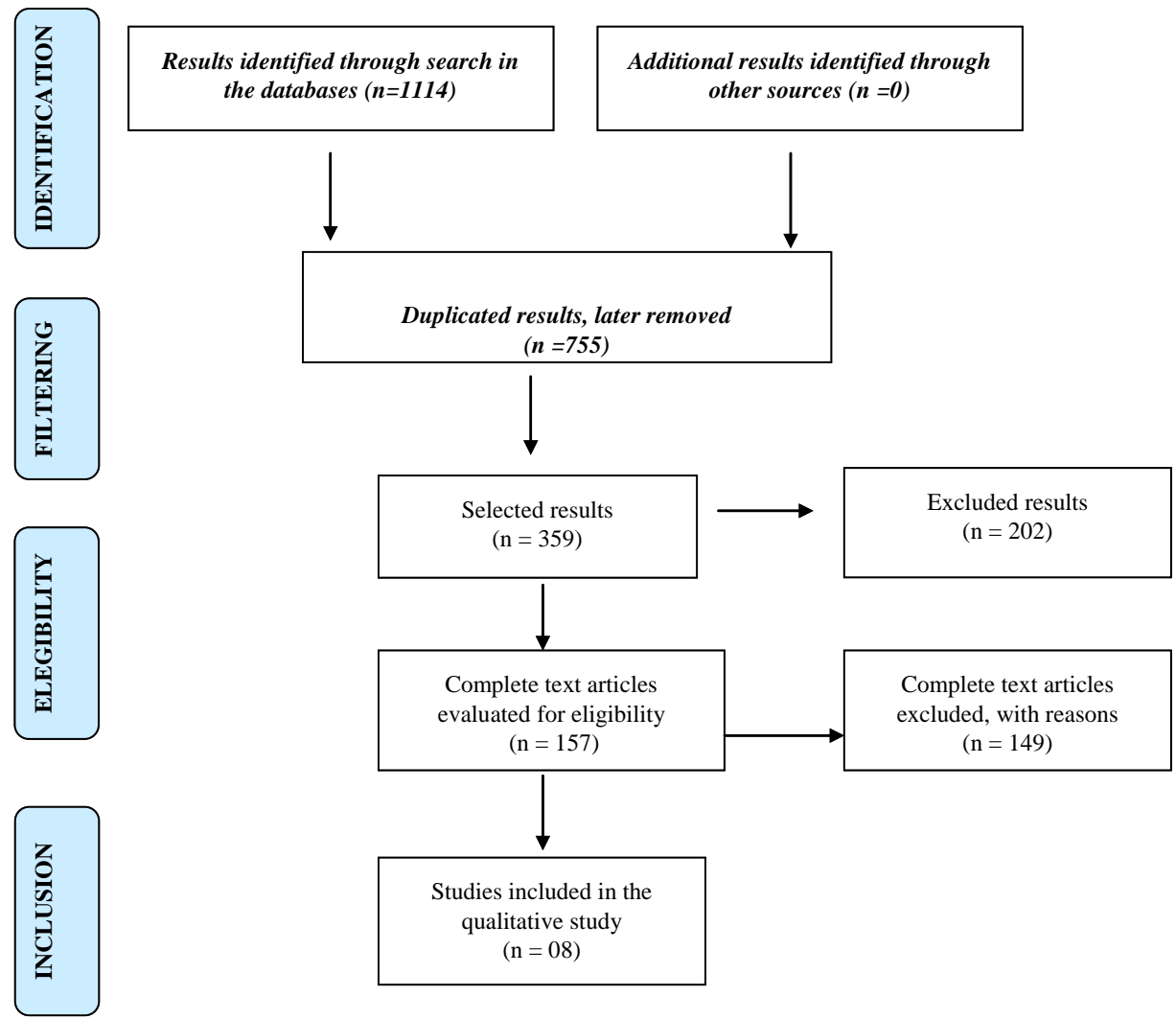

Figure 1. Identification process flowchart and article selection according to PRISMA Statemet, Moher et al., (2009) [14].

\subsection{Analysis and Synthesis}

The articles that were selected to compose this integrative review are described at Table 1. A descriptive summary of the results from the integrative review was generated. This summary table ware prepared for each primary study included in the review, including the following data: Authors, Year, Country of Origin, Level of Evidence, Study Design, Population, Items not contemplated in accordance with the STROBE tool and Main Results and Conclusions regarding ELS and Psychoactive Substances.

The date analysis of integrative review is descriptive. Our analysis will be presented in thematic categories: ELS and the Use of Alcohol; ELS and the Use of Psychoactive Substances during Adolescence; ELS and the Female Sex; ELS and Coping Strategies. This categories were identified after reading the studies.

This research was conducted according to the literature recommendations of Whittemore \& Knafl (2005) to conduct an Integrative Review (IR). The IR of the literature is a method of data collection that contributes to the phase of PBE [9] [12].

The eight articles selected were submitted to analysis to verify methods, and then relevant study information (appearance and content validation) was extracted with validated method by Ursi (2005), which contemplated the identification and methodological characteristics of the studies. The methodological course and the research design were analyzed with the aid of statistic. 
Table 1. Authors, Year, Country of Origin, Level of Evidence, Study Design, Population, Items not contemplated in accordance with the STROBE tool and Main Results and Conclusions regarding ELS and Psychoactive Substances.

(E1) Authors: Sarah F. Eames, Alina Suris, Uma Rao, Hong Xiao, Michael S. Businelle, Robrina Walker, Carol S. North and Bryon Adinoff.

Year: 2014

Title: Stress Moderates the Effect of Childhood Trauma and Adversity on Recent Drinking in

Treatment-Seeking Alcohol-Dependent Men.

Country of Origin: EUA

Keywords: Stress, Alcoholism, Childhood Trauma, Men, Trauma

Source: Journal of Consulting and Clinical Psychology

Level of Evidence: 2

Objective: This study sought to clarify the relationship between trauma/adversity in childhood with later alcohol consumption, and the moderating effects of psychosocial stress in adults.

Study Design: Quantitative cross-sectional study with observation, not experimentation, with no control group. Samples by convenience. Statistical analysis was performed using SPSS software and regression analysis. Significance level and type of tests are not specified in the analytical plan.

Population: Sample with 77 men recently in abstinence from alcohol who attend residential treatment programs.

STROBE: Does not contemplate: 1) Title and abstract: Provide in the abstract an informative and balanced summary of what was done and what was found; 9) Bias: Describe any efforts to address potential sources of bias; 17) Other analyses: Report other analyses done-eg analyses of subgroups and interactions, and sensitivity analyses; 22) Funding: Give the source of funding and the role of the funders for the present study and, if applicable, for the original study on which the present article is based.

Main Results and Conclusions: Childhood traumas predict the severity of drinking in alcohol dependent men, and this effect is stronger in participants with ongoing stress in adulthood. These findings suggest that trauma/adversity in childhood may sensitize the stress response systems [27].

(E2) Authors: Scott M. Hyman, Miguel Garcia and Rajita Sinha

Year: 2006

Title: Gender Specific Associations Between Types of Childhood Maltreatment and the Onset, Escalation and Severity of Substance Use in Cocaine Dependent Adults.

Country of Origin: EUA

Keywords: Childhood maltreatment; Gender Differences; substance Use

Source: Am J Drug Alcohol Abuse

Level of Evidence: 2

Objective: To examine the associations between the types of child maltreatment and its inception, climbing and severity of substance use in cocaine-dependent adults.

Study Design: Quantitative cross-sectional study with observation, not experimentation, with no control group. Samples by convenience. Software used for statistical analysis and significance level not informed the data analysis plan. Held simple regression analysis. Tests: Chi-square, T-test and Wilcoxon Rank-Sum. Population: Sample with 87 participants, men $(n=55)$ and women $(n=32)$ in treatment for cocaine addiction.

STROBE: Does not contemplate: 5) Setting: Describe the setting, locations, and relevant dates, including periods of recruitment, exposure, follow-up, and data collection; 6) Participants: Cross-sectional study-Give the eligibility criteria, and the sources and methods of selection of participants; 9) Bias: Describe any efforts to address potential sources of bias; 14) Descriptive data: (a) Give characteristics of study participants (eg demographic, clinical, social) and information on exposures and potential confounders; 19) Limitations: Discuss limitations of the study, taking into account sources of potential bias or imprecision. Discuss both direction and magnitude of any potential bias.

Main Results and Conclusions: In men, emotional abuse was associated with a younger age for the first use of alcohol and a bigger severity of substance abuse. In women, the sexual abuse, emotional abuse and mistreatment in general were associated with the first use of alcohol, emotional abuse, neglect and mistreatment were associated with a bigger severity of substance abuse. The results suggest that early intervention for childhood victims especially girls, may delay or prevent the early onset of alcohol use and reduce the risk of a more severe course of addiction [17]. 


\section{Continued}

(E3) Authors: Sunny Hyucksun Shin, Hyokyoung Grace Hong and Andrea L. Hazen

Year: 2010

Title: Childhood Sexual Abuse and Adolescent Substance Use: a latent class analysis.

Country of Origin: EUA

Keywords: Sexual abuse, Substance use, Adolescents, Childhood maltreatment, Latent class analysis, Physical abuse, Drug use, Child neglect

Source: Drug and Alcohol Dependence

Level of Evidence: 2

Objective: To characterize changes in substance use patterns in adolescents and to examine the association between childhood sexual abuse and qualitatively distinct patterns of substance use in adolescents.

Study Design: Quantitative cross-sectional study with observation, not experimentation, with no control group. Samples by convenience. Used SAS software for statistical analysis. Held multinomial logistic regression analysis, significance level of 5\%. Made Bayesian Information Criterion Test (BIC) and likelihood ratio test (LMR).

Population: Sample with 1,019 adolescents between the 13 and 18 years old (age average: 15.9 years) selected from five public funding systems.

Main Results and Conclusions: Child Sexual Abuse was associated to a higher risk for girls to develop Strong abuse of substances. The results indicate that women victims of childhood sexual abuse who are involved in public service systems, are at high risk of developing the use of multiple substances in adolescence.

STROBE: Does not contemplate items 9) Bias: Describe any efforts to address potential sources of bias; 11) Quantitative variables: Explain how quantitative variables were handled in the analyses. If applicable, describe which groupings were chosen and why; 13) Participants: 1) Report numbers of individuals at each stage of study—eg numbers potentially eligible, examined for eligibility, confirmed eligible, included in the study, completing follow-up, and analysed, 2) Give reasons for non-participation at each stage; 14) Descriptive data: 1) Give characteristics of study participants (eg demographic, clinical, social) and information on exposures and potential confounders, 2) Indicate number of participants with missing data for each variable of interest [18].

(E4) Authors: Adriana M. Tucci, Florence Kerr-Corrêa and Maria Lucia O. Souza-Formigoni

Year: 2010

Title: Childhood Trauma in Substance Use Disorder and Depression: an analysis by gender among a Brazilian clinical sample.

Country of Origin: Brazil

Keywords: Substance Use Disorder, Depression, Childhood Trauma, Gender, Emotional Abuse, Physical Abuse, Psychological abuse

Source: Child Abuse \& Neglect

Level of Evidence: 2

Objective: This study compared the frequency and intensity of childhood trauma in patients dependent on alcohol or other drugs in patients with depression, and a group control without psychiatric diagnoses.

Study Design: Quantitative cross-sectional study with observation, not experimentation, with the presence of a control group. Samples by convenience. Statistical software was used Statistica. Held Analysis Logistic regression. Tests: Chi-square, ANOVA and Newman-Keuls. Level of significance 5\%.

Population: Sample with 295 participants of both sexes. Divided into 03 groups (alcohol or other drugs dependent, patients with depression and control group with no psychiatric diagnosis).

Main Results and Conclusions: A higher frequency and intensity of emotional, physical and sexual abuse was found in patients dependent on alcohol or other drugs when compared to the group of depressed patients, which in turn show a bigger proportion than the control group. In all groups, higher frequency was found in the female sex. Because of the high frequency and intensity of childhood trauma among patients dependent on alcohol or other drugs and depressed patients, evaluation of problems due to childhood trauma among these patients is essential for a better understanding of the etiology of such diseases and their treatment.

STROBE: Does not contemplate items: 6) Paricipants: Cross-sectional study-Give the eligibility criteria, and the sources and methods of selection of participants; 7) Variables: Clearly define all outcomes, exposures, predictors, potential confounders, and effect modifiers. Give diagnostic criteria, if applicable; 19) Limitations: Discuss limitations of the study, taking into account sources of potential bias or imprecision. Discuss both direction and magnitude of any potential bias; 22) Funding: Give the source of 


\section{Continued}

funding and the role of the funders for the present study and, if applicable, for the original study on which the present article is based [21].

(E5) Authors: Jessica R. Peltan and Tony Cellucci

Year: 2011

Title: Childhood Sexual Abuse and Substance Abuse Treatment Utilization among Substance-Dependent Incarcerated Women.

Country of Origin: EUA

Keywords: Treatment utilization; Childhood sexual abuse; Incarcerated women

Source: Journal of Substance Abuse Treatment

Level of Evidence: 2

Objective: Incarcerated women have high rates of substance abuse problems and trauma. A large number of variables can influence these women are seeking help for the treatment of problems of substance abuse.

Study Design: Quantitative cross-sectional study with observation, not experimentation, with no control group. Samples by convenience. Significance level not informed in the method. Performed regression analysis. Testing:

T-test and correlation test.

Population: Sample with 40 participants, all of them in prison.

Main Results and Conclusions: The gravity of sexual abuse during childhood and current symptoms of trauma were negatively related to episodes of treatment for substance abuse. These women can use psychoactive substances as a means of coping childhood trauma or may not see substance abuse as responsive to co-occurrence symptoms of trauma.

STROBE: Does not contemplate items 1) Title and abstract: (b) Provide in the abstract an informative and balanced summary of what was done and what was found; 2) Background/rationale: Explain the scientific background and rationale for the investigation being reported; 4) Study design: Present key elements of study design early in the paper; 7) Variables: Clearly define all outcomes, exposures, predictors, potential confounders, and effect modifiers. Give diagnostic criteria, if applicable; 12) Statistical methods: Cross-sectional study-If applicable, describe analytical methods taking account of sampling strategy [19].

(E6) Authors: Meeyoung Min, Kathleen Farkas, Sonia Minnes and Lynn T. Singer

Year: 2007

Title: Impact of Childhood Abuse and Neglect on Substance Abuse and Psychological Distress in A dulthood.

Country of Origin: EUA

Keywords: No Contains

Source: Journal of Traumatic Stress

Level of Evidence: 2

Objective: To test the hypothesis that people with greater use of avoidant coping strategies are hypothetically susceptible to substance abuse and have serious levels of psychological distress.

Study Design: Quantitative cross-sectional study with observation, not experimentation, with no control group. Samples by convenience. Software used for statistical analysis was SPSS, AMOS module. Held Analysis of Structural Equation. Significance level of 5\%. Test: Pearson's correlation test.

Population: Sample of 285 women in post-partum in a teaching hospital.

Main Results and Conclusions: Childhood trauma were directly and indirectly related to psychological stress and substance abuse. The childhood trauma self-reported was significantly related to higher substance abuse and psychological disorders, through low levels of education and avoidant coping strategies. The results indicate importance of educational strategies to support and interventions to teach coping skills to prevent substance abuse and long-term psychological distress in children exposed to trauma.

STROBE: Does not contemplate items: 7) Variables: Clearly define all outcomes, exposures, predictors, potential confounders, and effect modifiers. Give diagnostic criteria, if applicable; 9) Bias: Describe any efforts to address potential sources of bias; 17) Other analyses: Report other analyses done-eg analyses of subgroups and interactions, and sensitivity analyses; 22) Funding: Give the source of funding and the role of the funders for the present study and, if applicable, for the original study on which the present article is based [20]. 


\section{Continued}

(E7) Authors: Luciana Burim Scomparini, Bernardo dos Santos, Robert Alan Rosenheck and Sandra Scivoletto

Year: 2013

Title: Association of Child Maltreatment and Psychiatric Diagnosis in Brazilian Children and Adolescents Country of Origin: Brazil

Palavras-Chave: Child Abuse; Psychiatric Disorders; Shelter; Community Programs; Follow-up Studies. Fonte: Clinics

Level of Evidence: 2

Objective: To evaluate the association between different types of abuse and the presence of psychiatric disorders in children and adolescents highly vulnerable, attended by a multidisciplinary program.

Study Design: Quantitative cross-sectional study with observation, not experimentation, with no control group. Samples by convenience. Statistical software used: SPSS. Held Analysis Logistic Regression with significance level of $5 \%$. Tests not informed.

Population: Sample of 351 participants, children and adolescents that have lived in shelters.

Main Results and Conclusions: The most commons psychiatric diagnosis found in the sample was the Disorder by Substance Use. All patients suffered from negligence and more than half experience physical or sexual abuse. Physical abuse and emotional neglect were most strongly associated with mental retar dation and the presence of multiple traumas related to substance abuse.

STROBE: Does not contemplate items: 3) Objectives: State specific objectives, including any prespecified hypotheses; 4) Study design: Present key elements of study design early in the paper; 6) Participants: Cross-sectional study-Give the eligibility criteria, and the sources and methods of selection of participants; 7) Variables: Clearly define all outcomes, exposures, predictors, potential confounders, and effect modifiers. Give diagnostic criteria, if applicable; 11) Quantitative variables: Explain how quantitative variables were handled in the analyses. If applicable, describe which groupings were chosen and why; 14) Descriptive data: (a) Give characteristics of study participants (eg demographic, clinical, social) and information on exposures and potential confounders, (b) Indicate number of participants with missing data for each variable of interest; 17) Other analyses: Report other analyses done-e.g. analyses of subgroups and interactions, and sensitivity analyses [22].

(E8) Authors: Marc Vogel, Kenneth M. Dürsteler-MacFarland, Marc Walter, Johannes Strasser, Stephanie Fehr, Luis Prieto and Gerhard A. Wiesbeck

Year: 2011

Title: Prolonged use of Benzodiazepines is Associated with Childhood Trauma in Opioid-Maintained Patients.

Country of Origin: Switzerland

Keywords: Sedative, Diazepam, Adverse childhood events, Opioid dependence

Source: Drug and Alcohol Dependence

Level of Evidence: 2

Objective: Abusive use of Benzodiazepines (BZD) in Opioid users in treatment is very common and has been linked to poor and unsuccessful treatments. This study aims to examine the possibility of traumatic experiences in childhood in patients treated with oral or injectable diacetylmorphine opioids.

Study Design: Quantitative cross-sectional study with observation, not experimentation, with no control group. Samples by convenience. Statistical software used: SPSS and STATA. Performed logistic regression analysis.

Significance level of 5\%. Tests: Cohen's kappa, chi-square, Fisher, Mann-Whitney and Kendall tau-b. Population: Sample with 193 participants, BZD users in treatment due to the use of opioids.

Main Results and Conclusions: The use of BZD was predominant and the load of childhood traumatic experiences was high, with $67 \%$ reporting having suffered at least one type of trauma in a moderate or severe way. Traumatic experiences in childhood may be associated with prolonged use of BZD and may represent an important starting point for prevention.

STROBE: Does not contemplate items 3) Objectives: State specific objectives, including any prespecified hypotheses; 9) Bias: Describe any efforts to address potential sources of bias; 14) Descriptive data: 1) Give characteristics of study participants (eg demographic, clinical, social) and information on exposures and potential confounders; 2) Indicate number of participants with missing data for each variable of interest [25]. 
The level of strength of evidence of the studies was analyzed according to the PBE criteria, using the Evidence Classification [10]. All the studies included have level-2 evidence strength (evidence derived from a single study of cut or case-control study) according to the Evidence Classification to studies of clinical nature of Prognosis/Prediction or Etiology.

With the purpose of discussing the quality of each study selected, the STROBE Statement was used. It is a checklist composed of 22 items with recommendations, with the purpose of guiding authors regarding content to be contemplated in observational studies, seeking a more complete description to improve the quality of the scientific article [13].

\section{Discussion}

\subsection{ELS and the Use of Alcohol}

The psychoactive drug more often used by those participating in these selected studies for this review was alcohol. Individuals tend to start with alcohol and nicotine (legal substances) before starting with illegal substances. According with the IV National Survey regarding Psychotropic Drugs among Students of Public and Private Middle School and High School in the 27 Brazilian Capitals in 2010, alcohol and tobacco are the prevalent drugs used in life, in all capitals, followed by inhalant drugs [15].

Cigarettes and alcoholic beverages, mainly beer and wine, were indicated as having been used much earlier in life than illicit drugs. On average, marijuana tends to come 2, 5 years after the use of cigarettes or alcoholic beverages, and cocaine and crack were used a little after a year from marijuana [16].

The age of the first use of alcohol is considered as a predictor for the age of cocaine first use in both genders. It may have indirect correlation to mistreatment during childhood and the leaning towards using cocaine, which is predicted using the first use of alcohol. Childhood mistreatment may contribute directly to the age of first use of alcohol. The use of alcohol and nicotine opens the door to other drugs [17].

In the study by Shin et al. (2010) [18], which classified adolescent substance users in latent classes, the use of alcohol and cannabis was present in all classes of substance users, and all the classes which made use of cannabis, also reported having used alcohol as well. All classes that used heavy drugs reported also having used alcohol and cannabis.

In the Peltan \& Cellucci (2011) study, 55\% of the sample reported the use of alcohol while consuming other drugs. In the Min et al. (2007) study, $88 \%$ of the sample of pre-natal mothers mad use of at least one substance during pregnancy, while over half (66\%) made use of alcohol. Alcohol had a significant connection with 4 of the 5 types of trauma, not being linked, however, with Emotional Abuse, in the study performed by Tucci et al. (2010) [19] [20] [21].

\subsection{ELS and the Use of Psychoactive Substances during Adolescence}

Mistreatment and cumulative stressful events before puberty and especially in the first years of life is linked to the early drinking abuse in adolescence and alcohol and other 
drugs dependence at the beginning of adulthood [5].

In Scomparini et al. (2013), a study performed with children and adolescents with an average of 12.47 years, the Substance Use Disorder was positively associated with the exposure to multiple traumas and with each new trauma, the likelihood of the disorder increased by $34 \%$ [22].

Shin et al. (2010), defend that adolescents involved in public service in the city (regardless of sex), when older, are more likely to use multiple substances when compared to younger adolescents (ages between 13 - 15 years). The bigger risk factor is the influence of use by pairs and the protection factor is the parental control [18].

In the study by White et al. (2013), adolescents that made use of multiple substances were directly associated with psychological stress and use by pairs. Older adolescents tended to have higher access and availability throughout life to a myriad of drugs when compared to younger adolescents [23].

While mistreatment during childhood may contribute directly to the age of first alcohol use, other factors in the early use of alcohol in the adolescent's life (for example, the use of substances by pairs) may contribute to a faster progression of cocaine use [17].

\subsection{ELS and the Female Sex}

Authors who have performed research with both gender, indicate that childhood traumas and later use of psychoactive substances are more present in female [17] [18] [21].

The ELS may affect directly upon the vulnerability of women regarding the use of alcohol, especially in association with stress factors beyond their control. Extremely stressful life events were associated with the consumption of alcohol only among women and these effects were negative on men, when exposed to mistreatment during childhood [24].

Scomparini et al. (2013), observed a higher average of adverse events among women, when compared to men, and Vogel et al. (2011), observed that women were more likely to obtain higher scores in the CTQ, higher subscores of moderate or serious Emotional Abuse, Physical Abuse and Sexual Abuse [23] [24].

In Hyman et al. (2006), Sexual Abuse, Emotional Abuse and Mistreatment in General were strongly linked with the age at which women dependent on cocaine started first using alcohol, and the age of the first use of alcohol was directly associated with the age cocaine would first be used and used regularly throughout life. Emotional Abuse, Emotional Neglect and Mistreatment in General were positively associated with the use of substance throughout life, and Sexual Abuse was strongly associated with the use of alcohol for the first time by women [17].

According to Shin et al. (2010), women who suffered sexual abuse are more likely to use multiple substances in adolescence (this association was negative in male) [18].

In the study performed by Tucci et al. (2010), the frequency and intensity of Emotional Abuse, Physical Abuse and Sexual Abuse in patients who have alcohol and/or other drugs dependency was higher in women than in men [21]. 
Heffner et al. (2011), conducted a study with both gender regarding the gravity of the childhood traumas and how it could be associated with relapses in the use of alcohol and/or other psychoactive substances with women but not with men [26].

Another study performed by Hyman et al. (2008), also had results showing that childhood trauma increased the chance of relapse in the use of psychoactive substances only with women $[2]$.

\subsection{ELS and Coping Strategies}

A study with incarcerated women showed a negative association between Sexual Abuse and other traumas regarding the treatment for psychoactive substances abuse. This abusive substance use may have been used as a coping mechanism (non-assertive coping), or these women failed to notice the connection between the substance abuse and the trauma lived [19].

Childhood trauma were significantly associated with low levels of education and use of non-assertive coping mechanisms (avoidance). The reason for this low level of education may be due to Neglect and Emotional Abuse of children that have not received the proper parental support to attend school regularly and thus learned to use avoidance tactics, such as skipping class and using alcohol and other drugs to deal with the impact of the abuse and/or neglect [20].

For Eames, et al. (2014), the association between ELS and the later use of alcohol may be due to traumas, which conditioned the way the child learned to deal with stress and can predict the gravity of alcohol dependence. This effect was stronger on men that have lived with stress continuously throughout their adult lives. Due to stress factors during rehabilitation, individuals in treatment who experienced high levels of childhood trauma are more likely to relapse [27].

Mistreatment during childhood may potentialize the genetic vulnerability to alcohol use as a stress coping mechanism, and the impact of these effects may be measured by the interaction between gene and environment. Risk factors such as childhood mistreatment likely increase the change these individuals have of developing non-assertive coping mechanisms [5] [24].

\subsection{Nursing Contribution on ELS}

Considering the nursing mental health care developed during the vital cycle, nurses represent professionals that are remaining most time with patients. These professionals provide assistance to children and adolescents, and have the better opportunities to observe and identify the signs and symptoms of abuse and neglect in childhood, which means ELS. Nursing professionals should act preventively about ELS in nursing care plan. In this way, nurses have to provide to their clients possibilities to develop assertive coping strategies and even actuate legal support for removal of stress factors when it would be necessary.

Among adults who suffered ELS and developed problematic use of psychoactive substances, the ELS should be identified, and stress reduction interventions must be guar- 
anteed, considering a cumulative history of adversities throughout life.

\section{Study Limitations}

The studies included in this review corroborate the research question, showing that the use and abuse of psychoactive substances in adolescence and adulthood is an outcome of the childhood trauma, though each one should be carefully assessed.

All studies included in our sample are cross-sectional studies, so there may be casual interference, and it is known that other factors may also influence drug use (genetics, family, social factors etc.) (Hyman et al. 2006; Maltreatments experienced in childhood are self-reported, so there may be memory leaks or omission of facts. The samples of studies was gathered by convenience (they were already users of alcohol and/or other psychoactive substances, or were considered a population at risk/vulnerable) [17] [28].

In our sample, only one study was conducted in Brazil by Tucci et al. (2010) with a control group (psychoactive substances non-dependent group without psychiatric diagnosis), confirming the hypothesis that childhood trauma was prevalent in the group that made use of psychoactive substances [21].

The studies used different types of data collection for evaluating the use of psychoactive substances and to measure other psychiatric disorders, but all the authors selected for this review used only the CTQ as a tool to assess ELS.

The research associated with Childhood Trauma is an interdisciplinary field, since the children's wellbeing is a concern not only for health care courses, but to the education, social services, legal fields, as well as for the public in general. Despite being a multi/interdisciplinary field, there was not found studies performed by nurses to answer the research question, even if the search was performed in multidisciplinary databases [29].

\section{Conclusions and Implications}

Through this integrative literature review, scientific evidence supporting ELS as one of the factors of alcohol abuse and/or other psychoactive substances in adolescence and adulthood were identified in all articles included in this study. Studies show that the occurrence of mistreatment in combination with environmental and genetic factors influence the onset of substance use, the emergence and maintenance of addiction and also the relapse processes.

As a drug, alcohol was the most frequent substance in this research. In addition to alcohol and other illicit drugs, traumatic childhood experiences may also be associated with prolonged use of BZD in patients undergoing treatment for opioid use and this may be an important starting point for the prevention of substances abuse [25].

ELS may turn adolescents living in populations at risk more vulnerable to alcohol and other psychoactive substances. The improvement of substance use prevention and the creation of treatment services for adolescents, victims of Child Sexual Abuse is imperative. It is important that appropriate public policies be developed for the prevention of child abuse, especially to reduce the risk of subsequent mental disabilities [18] 
[22].

In studies using samples with both genders, there is the confirmation that the abuse of psychoactive substances after ELS, was more frequent in women. The early interventions for abused children, especially females, can help delay or prevent the early onset of alcohol use and reduce the risk of a more severe dependence [17].

Min et al. (2007) suggests the importance of understanding trauma in women seeking treatment for psychological disorders and substance abuse, so that interventions may be created to promote assertive coping skills, to reduce or prevent the problems associated with childhood trauma [20].

Incarcerated women who suffered ELS have shown low levels of demand for treatment for the abuse of psychoactive substances. One should consider and seek to understand the barriers faced by this population, so that better interventions can be provided to encourage them to seek help [19].

It was also possible to identify the use of psychoactive substances as a non-assertive coping strategy for traumas experienced in childhood, and in addition to the use of psychoactive substances, ELS is strongly linked with the development of psychological stress disorders. It is important to include traumas in childhood in the diagnostic process of people making treatment for substance abuse, so these coping strategies can be worked, reducing the number of relapses [21]. Eames et al. (2014), suggests a strong need to work on these coping skills, stress management background and pharmacological treatment at the beginning of the recovery process for substances users [27].

The author used CTQ only as a tool to measure ELS and confirmed it as being highly useful to identify childhood traumas.

This study reinforces the need to investigate the ELS occurrence in people that abuse of psychoactive substances, as strategy to improve treatment, making it more effective for these specific patients. Assertive coping skills must be developed, as well as stress management. It is important to note that early interventions with mistreated children (especially females) helps to slow down or even prevent substance abuse later on and a more serious evolution of psychoactive substances dependence.

\section{References}

[1] Lipp, M.E.N. (2001) Estresse Emocional: A Contribuição de Estressores Internos e Externos. Archives of Clinical Psychiatry, 28, 347-349.

[2] Hyman, S.M., Paliwal, P., Chaplin, T.M., Mazure, C.M., Rounsaville, B.J. and Sinha, R. (2008) Severity of Childhood Trauma Is Predictive of Cocaine Relapse Outcomes in Women but Not Men. Drug and Alcohol Dependence, 92, 208-216. http://dx.doi.org/10.1016/j.drugalcdep.2007.08.006

[3] Afifi, T.O., Mota, N.P., Dasiewicz, P., MacMillan, H.L. and Sareen, J. (2012) Physical Punishment and Mental Disorders: Results from a Nationally Representative US Sample. Pediatrics, 130, 1-9. http://dx.doi.org/10.1542/peds.2011-2947

[4] Bernstein, D.P., Stein, J.A., Newcomb, M.D., Walker, E., Pogge, D., Ahluvalia, T., et al. (2003) Development and Validation of a Brief Screening Version of the Childhood Trauma Questionnaire. Child Abuse \& Neglect, 27, 169-190. http://dx.doi.org/10.1016/S0145-2134(02)00541-0 
[5] Enoch, M.A. (2012) The Influence of Gene-Environment Interactions on the Development of Alcoholism and Drug Dependence. Current Psychiatry Reports, 14, 150-158. http://dx.doi.org/10.1007/s11920-011-0252-9

[6] Blefari, A.L. (2002) A família e a drogadição. Monografia de Especialização, Faculdade de Medicina, Universidade de São Paulo, São Paulo.

[7] Bernstein, D.P., Ahluvalia, T., Pogge. D. and Handelsman, L. (1997) Validity of the Childhood Trauma Questionnaire in an Adolescent Psychiatric Population. Journal of the American Academy of Child and Adolescent Psychiatry, 36, 340-348. http://dx.doi.org/10.1097/00004583-199703000-00012

[8] Grassi-Oliveira, R., Stein, L.M. and Pezzi, J.C. (2006) Tradução e validação de conteúdo da versão em português do Childhood Trauma Questionnaire. Revista de Saúde Pública, 40, 249-255. http://dx.doi.org/10.1590/S0034-89102006000200010

[9] Beyea, S.C. and Nicoll, L.H. (1998) Writing an Integrative Review. AORN Journal, 67, 877880. http://dx.doi.org/10.1016/S0001-2092(06)62653-7

[10] Melnyk, B.M. and Fineout-Overholt, E. (2011) Making the Case for Evidence-Based Practice. In: Melnyk, B.M. and Fineout-Overholt, E., Eds., Evidence-Based Practice in Nursing \& Healthcare. A Guide to Best Practice. 2nd Edition, Lippincot Williams \& Wilkins, Philadelphia, 3-24.

[11] Mendes, K.S., Silveira, R.C.C.P. and Galvão, C.M. (2008) Revisão integrativa: Método de pesquisa para a incorporação de evidências na saúde e na enfermagem. Texto \& Contexto Enfermagem, 17, 758-764. http://dx.doi.org/10.1590/S0104-07072008000400018

[12] Whittemore, R. and Knafl, K. (2005) The Integrative Review: Updated Methodology. Journal of Advanced Nursing, 52, 546-553. http://dx.doi.org/10.1111/j.1365-2648.2005.03621.x

[13] Elm, V.E., Altman, D.G., Egger, M., Pocock, S.J., Gotzsche, P.C. and Vandenbroucke, J.P., STROBE Initiative (2008) The Strengthening the Reporting of Observational Studies in Epidemiology (STROBE) Statement: Guidelines for Reporting Observational Studies. Journal of Clinical Epidemiology, 61, 344-349. http://dx.doi.org/10.1016/j.jclinepi.2007.11.008

[14] Moher, D., Liberati, A., Tetzlaff, J. and Altman, D.G., The PRISMA Group (2009) Preferred Reporting Items for Systematic Reviews and Meta-Analyses: The PRISMA Statement. PLoS Medicine, 6, e1000097. http://dx.doi.org/10.1371/journal.pmed.1000097

[15] Carlini, E.L.A., Noto, A.R., Sanchez, Z.M., Carlini, C.M.A., Locatelli, D.P., Abeid, L.R., et al. (2010) IV Levantamento nacional sobre o consumo de drogas psicotrópicas entre estudantes do ensino fundamental e médio das redes pública e privada de ensino nas 27 capitais brasileiras. Centro Brasileiro de Informações sobre Drogas Psicotrópicas; Universidade Federal de São Paulo; Brasília: Secretaria Nacional de Políticas sobre Drogas, São Paulo.

[16] Kandel, D. and Yamaguchi, K. (1993) From Beer to Crack: Developmental Patterns of Drug Involvement. American Journal of Public Health, 83, 851-855.

http://dx.doi.org/10.2105/AJPH.83.6.851

[17] Hyman, S.M., Garcia, M. and Sinha, R. (2006) Gender Specific Associations between Types of Childhood Maltreatment and the Onset, Escalation and Severity of Substance Use in Cocaine Dependent Adults. American Journal of Drug and Alcohol Abuse, 32, 655-664. http://dx.doi.org/10.1080/10623320600919193

[18] Shin, S.H., Hong, H.G. and Hazen, A.L. (2010) Childhood Sexual Abuse and Adolescent Substance Use: A Latent Class Analysis. Drug and Alcohol Dependence, 109, 226-235. http://dx.doi.org/10.1016/j.drugalcdep.2010.01.013

[19] Peltan, J.R. and Cellucci, T. (2011) Childhood Sexual Abuse and Substance Abuse Treatment Utilization among Substance-Dependent Incarcerated Women. US. Journal of Sub- 
stance Abuse Treatment, 41, 215-224. http://dx.doi.org/10.1016/j.jsat.2011.03.004

[20] Min, M., Farkas, K., Minnes, S. and Singer, L.T. (2007) Impact of Childhood Abuse and Neglect on Substance Abuse and Psychological Distress in Adulthood. Journal of Traumatic Stress, 20, 833-844. http://dx.doi.org/10.1002/jts.20250

[21] Tucci, A.M., Kerr-Corrêa, F. and Souza-Formigoni, M.L. (2010) Childhood Trauma in Substance Use Disorder and Depression: An Analysis by Gender among a Brazilian Clinical Sample. Child Abuse \& Neglect, 34, 95-104. http://dx.doi.org/10.1016/j.chiabu.2009.07.001

[22] Scomparini, L.B., Santos, B., Rosenheck, R.A. and Scivoletto, S. (2013) Association of Child Maltreatment and Psychiatric Diagnosis in Brazilian Children and Adolescentes. Clinics, 68, 1096-1102. http://dx.doi.org/10.6061/clinics/2013(08)06

[23] White, A., Chan, G.C.K., Quek, L.-H., Connor, J.P., Saunders, J.B., Baker. P., et al. (2013) The Topography of Multiple Drug Use among Adolescent Australians: Findings from the National Drug Strategy Household Survey. Addictive Behaviors, 38, 2068-2073. http://dx.doi.org/10.1016/j.addbeh.2013.01.001

[24] Young-Wolff, E.C., Kendler, K.S. and Prescott, C.A. (2012) Interactive Effects of Childhood Maltreatment and Recent Stressful Life Events on Alcohol Consumption in Adulthood. Journal of Studies on Alcohol and Drugs, 73, 559-569. http://dx.doi.org/10.15288/jsad.2012.73.559

[25] Vogel, M., Dürsteler-Macfarland, K.M., Walter, M., Strasser, J., Fehr, S., Prieto, L., et al. (2011) Prolonged Use of Benzodiazepines Is Associated with Childhood Trauma in OpioidMaintained Patients. Drug and Alcohol Dependence, 119, 93-98. http://dx.doi.org/10.1016/j.drugalcdep.2011.05.037

[26] Heffner, J.L., Blom, T.J. and Anthenelli, R.M. (2011) Gender Differences in Trauma History and Symptoms as Predictors of Relapse to Alcohol and Drug Use. American Journal on Addictions, 20, 307-311. http://dx.doi.org/10.1111/j.1521-0391.2011.00141.x

[27] Eames, S.F., Suris, A., Rao, U., Xiao, H., Businelle, M.S., Walker, R., et al. (2014) Stress Moderates the Effect of Childhood Trauma and Adversity on Recent Drinking in Treatment-Seeking Alcohol-Dependent Men. Journal of Consulting and Clinical Psychology, 82, 441-447. http://dx.doi.org/10.1037/a0036291

[28] Fenton, M.C., Geier, T., Keyes, K., Skodol, A.E., Grant, B.F. and Hasin, D.S. (2013) Combined Role of Childhood Maltreatment, Family History, and Gender in the Risk for Alcohol Dependence. Psychological Medicine, 43, 1045-1057. http://dx.doi.org/10.1017/S0033291712001729

[29] Mulvihill, D. (2005) The Health Impact of Childhood Trauma: An Interdisciplinary Review, 1997-2003. Issues in Comprehensive Pediatric Nursing, 28, 115-136. http://dx.doi.org/10.1080/01460860590950890 
Submit or recommend next manuscript to SCIRP and we will provide best service for you:

Accepting pre-submission inquiries through Email, Facebook, LinkedIn, Twitter, etc. A wide selection of journals (inclusive of 9 subjects, more than 200 journals)

Providing 24-hour high-quality service

User-friendly online submission system

Fair and swift peer-review system

Efficient typesetting and proofreading procedure

Display of the result of downloads and visits, as well as the number of cited articles

Maximum dissemination of your research work

Submit your manuscript at: http://papersubmission.scirp.org/

Or contact ojn@scirp.org 